\title{
A NOTE ON THE UNIQUENESS OF POSITIVE SOLUTIONS OF ROBIN PROBLEM*
}

\author{
QIUYI DAI \\ Department of Mathematics, Hunan Normal University, Changsha, Hunan 410081, P.R. China \\ e-mail:daiqiuyi@yahoo.com.cn \\ and YUXIA FU \\ Department of Applied Mathematics, Hunan University, Changsha, Hunan 410082, P.R. China
}

(Received 5 January 2007; accepted 24 May 2007)

\begin{abstract}
This note is devoted to prove some uniqueness results of positive solutions of a Robin problem for semi-linear elliptic equations and systems.
\end{abstract}

2000 Mathematics Subject Classification. 35J25, 35J55.

1. Introduction. Let $\Omega \subset R^{n}$ be a bounded smooth domain, $\alpha$ and $\beta$ be nonnegative real numbers and $p \in(1,(n+2) /(n-2))$. We consider the following problem:

$$
\begin{cases}\Delta u+u^{p}=0, & x \in \Omega, \\ u>0, & x \in \Omega, \\ \alpha \frac{\partial u}{\partial v}+\beta u=0, & x \in \partial \Omega\end{cases}
$$

where $v$ is the unit outer normal vector field on $\partial \Omega$.

When $\alpha>0$ and $\beta=0$, it is trivial to observe that (1.1) has no solution. On the other hand, if $\beta>0$, it is easy to prove via the variational method or the continuity method combined with a priori estimates that (1.1) has at least one solution. Since the existence of multiple solutions is a common phenomenon in the non-linear problem, a natural question then follows.

Question A: How many solutions does (1.1) have? In the Dirichlet case (that is, $\alpha=0$ and $\beta>0$ ), the answers to Question A depend on the shape of the domain. For example, (1.1) with $\alpha=0$ and $\beta>0$ has at most one solution when $\Omega$ is a ball or a planner domain, that is, symmetric and convex along axes (see [1, 6]). However, it has more than one solution for some dumbell-shaped domains with a narrow enough neck (see $[7,8]$ ), or some annulus domains. There are many other literatures on the Dirichlet problem. We refer the interest reader to $[3,8,10,15,16,17]$. Compared with the Dirichlet problem, there are few results about the so-called Robin problem (that is, $\alpha, \beta>0$ ) that always arise in biology, chemistry and environmental sciences (see, however, [9] for an existence result for the Robin problem with critical non-linearities). In this paper, we study the Robin problem. For convenience, we set $\eta=\beta / \alpha$ and

*This work is supported by the NNSF of China (No. 10671064). 
transform (1.1) into the following problem:

$$
\begin{cases}\Delta u+u^{p}=0, & x \in \Omega, \\ u>0, & x \in \Omega, \\ \frac{\partial u}{\partial v}+\eta u=0, & x \in \partial \Omega .\end{cases}
$$

Existence result for (1.2) can be proved in a similar way as that of [9]. Hence, our particular attention is given to prove uniqueness results for (1.2). In the Dirichlet case, it is well known that the symmetry and convexity of the domain play a crucial role in the proof of uniqueness results via a so-called maximum principle for narrow domains (see $[6,14])$. However, in the Robin boundary case, this kind of maximum principle is no longer available. The lack of maximum principle for narrow domains causes some difficulty in the analysis of problem (1.2). For example, we do not know how to prove a symmetrical result for (1.2) even when $\Omega$ is a ball, let alone $\Omega$ are other symmetrical domains. This circumstance prevents us from adopting many powerful tools used in the proof of uniqueness results for the Dirichlet problem. The method we will use here is the a priori estimates method combined with the analysis of the asymptotic behaviour of solutions of (1.2) as $\eta \rightarrow 0$. There is a drawback as well as an advantage of this method. The drawback is that we can obtain only local in $\eta$ results by this method. The advantage is that this method also works well for the Robin problem of elliptic systems. Now, we are in a position to state our first result.

THEOREM 1.1. There exists a positive number $\eta_{0}$ such that problem (1.2) has at most one solution for any $\eta \in\left(0, \eta_{0}\right)$.

Our second result is about the Robin problem of elliptic systems. To simplify the notation, we denote $\frac{\partial u}{\partial v}+\eta u$ by $B u$. Then the problem we consider next can be written as

$$
\begin{cases}\Delta u+v^{p}=0, & x \in \Omega \\ \Delta v+u^{q}=0, & x \in \Omega \\ u, v>0, & x \in \Omega \\ B u=B v=0, & x \in \partial \Omega\end{cases}
$$

THEOREM 1.2. There exists a positive number $\eta_{0}$ such that problem (1.3) has at most one solution for any $\eta \in\left(0, \eta_{0}\right)$ provided that $p, q \in(1,(n+2) /(n-2))$ or $\max \{2(p+$ $1) /(p q-1), 2(q+1) /(p q-1)\}>n-2$.

REMARK 1.3. Compared with the uniqueness results for a Dirichlet problem, the main feature of our results is that there is no special request on the domain $\Omega$.

REMARK 1.4. A difference between the Dirichlet problem and the Robin problem is also displayed in the symmetrical results. It is well known that the symmetrical results may be invalid for positive solutions of the Dirichlet problem on annulus domains. However, by Theorems 1.1 and 1.2, we have the following symmetrical results for the Robin problem when $\eta$ is small enough.

COROLLARY 1.5. If $\Omega$ is invariant under rotation, then, for any $\eta \in\left(0, \eta_{0}\right)$, the solution of problems (1.2) and (1.3) is radially symmetry, where $\eta_{0}$ is the positive number in Theorems 1.1 and 1.2 , respectively. 
REMARK 1.6. An interesting question is that whether the symmetrical result is valid for positive solutions of the Robin problem when $\eta>0$ is arbitrary and $\Omega$ is invariant under rotation.

The rest of this paper is arranged as follows. Section 2 includes some preliminary lemmas needed in the proof of Theorems 1.1 and 1.2. The proof of Theorems 1.1 and 1.2 is placed in Section 3.

2. Preliminary lemmas. In this section, we give some lemmas needed in the proof of Theorems 1.1 and 1.2.

LEMMA $2.1([4])$. Let $u(x)$ be a non-negative solution of the equation

$$
-\Delta u=u^{p}, \quad x \in R^{n},
$$

with $1 \leq p<\frac{n+2}{n-2}$, then $u(x) \equiv 0$.

LEMMA $2.2([\mathbf{2}, \mathbf{1 1}, \mathbf{1 2}])$. Let $(u(x), v(x))$ be a non-negative solution of the elliptic system

$$
\begin{cases}-\Delta u=v^{p}, & x \in R^{n} \\ -\Delta v=u^{q}, & x \in R^{n}\end{cases}
$$

with $p, q \in(1,(n+2) /(n-2))$ or $\max \{2(p+1) /(p q-1), 2(q+1) /(p q-1)\}>n-2$. Then $u(x) \equiv v(x) \equiv 0$.

LEMMA 2.3. There exists a positive number $\eta_{0}$ and a positive constant $M$ independent of $\eta$ such that for any solution $u(x)$ of problem (1.2) with $\eta \in\left(0, \eta_{0}\right)$, we have $\|u\|_{L^{\infty}(\bar{\Omega})} \leq$ $M$.

Proof. If the conclusion of Lemma 2.3 is not true, then there exists a sequence $\eta_{k} \rightarrow 0$ as $k \rightarrow \infty$ and a sequence of solution $u^{k}(x)$ of problem (1.2) with $\eta=\eta_{k}$ such that

$$
M_{k}=\max _{x \in \bar{\Omega}} u^{k}(x)=u^{k}\left(x_{k}\right) \rightarrow+\infty, \text { as } k \rightarrow \infty .
$$

Let $d_{k}=\operatorname{dist}\left(x_{k}, \partial \Omega\right)$ and $v_{k}$ be the function defined by

$$
v_{k}(y)=\frac{u^{k}\left(x_{k}+M_{k}^{(1-p) / 2} y\right)}{M_{k}}=\frac{u^{k}(x)}{M_{k}}, y=M_{k}^{(p-1) / 2}\left(x-x_{k}\right) .
$$

It is easy to check that $v_{k}$ satisfies

$$
\begin{cases}-\Delta v_{k}=v_{k}^{p}, & y \in \Omega_{k}, \\ \max _{y \in \Omega_{k}} v_{k}(y)=v_{k}(0)=1, & \\ \frac{\partial v_{k}(y)}{\partial v}+\eta_{k} v_{k}=0, & y \in \partial \Omega_{k},\end{cases}
$$

where $\Omega_{k}=M_{k}^{(p-1) / 2}\left(\Omega-x_{k}\right)$.

Now, we have the following two cases to be considered.

Case 1: If $d_{k} M_{k}^{(p-1) / 2} \rightarrow \infty$, then $\Omega_{k} \rightarrow R^{n}$. Hence, for any compact domain $D \subset$ $R^{n}$, we have $D \subset \Omega_{k}$ when $k$ is large enough. By the standard elliptic estimates, we 
can find a constant $C$ depending only on $D$ and $n$ such that $\left\|v_{k}\right\|_{C^{k, \alpha(D)}} \leq C$. If the diagonal convergence method is employed, then we can extract a subsequence of $v_{k}$ that is still denoted by $v_{k}$ such that $v_{k}$ converges uniformly on any compact subset of $R^{n}$ to a function $v$. Considering the limit in problem (2.3), we find that $v$ satisfies

$$
\left\{\begin{array}{l}
-\Delta v=v^{p}, \quad y \in R^{n} \\
v(0)=1
\end{array}\right.
$$

This contradicts the conclusion of Lemma 2.1.

Case 2: If $d_{k} M_{k}^{(p-1) / 2}$ is bounded, then we can extract a subsequence of $d_{k} M_{k}^{(p-1) / 2}$ that is still denoted by $d_{k} M_{k}^{(p-1) / 2}$ such that $d_{k} M_{k}^{(p-1) / 2} \rightarrow s$, as $k \rightarrow \infty$. In this circumstance, we have $\Omega_{k} \rightarrow R_{s}^{+}=R^{n} \cap\left\{x_{n}>-s\right\}$. Hence, for any compact domain $D$ of $R_{s}^{+}$, it follows from the standard elliptic estimates that there exists a constant $C$ depending only on $D$ and $n$ such that $\left\|v_{k}\right\|_{C^{2, \alpha}(D)} \leq C$. By the diagonal convergence method, we know that up to a subsequence, $v_{k}$ converges uniformly on any compact subset of $R_{s}^{+}$to a function $v(y)$. Considering the limit in problem (2.3), we find that $v(y)$ satisfies

$$
\begin{cases}-\Delta v=v^{p}, & y \in R_{s}^{+}, \\ v(0)=1, & \\ \frac{\partial v}{\partial y_{n}}=0, & y_{n}=-s .\end{cases}
$$

If we denote the even reflection of $v(y)$ with respect to the hyperplane $y_{n}=-s$ by $w(y)$, and set

$$
h(y)= \begin{cases}v(y), & y \in \overline{R_{s}^{+}}, \\ w(y), & y \in R_{s}^{-}=\left\{y \in R^{n}: y_{n}<-s\right\},\end{cases}
$$

then it is easy to verify that $h(y)$ is a non-trivial solution of problem (2.1). This also contradicts the conclusion of Lemma 2.1. Hence, we complete the proof of Lemma 2.3.

By a similar argument to that used in the proof of Lemma 2.3 and taking into account Lemma 2.2, we can prove the following lemma (a similar argument can be found in [11]).

LEMMA 2.4. There exists a positive number $\eta_{0}$ and a positive constant $M$ independent of $\eta$ such that for any solution $(u(x), v(x))$ of problem (1.3) with $\eta \in\left(0, \eta_{0}\right)$, we have

$$
\|u\|_{L^{\infty}(\bar{\Omega})}+\|v\|_{L^{\infty}(\bar{\Omega})} \leq M .
$$

LEMMA 2.5. Let $u_{1}(x)$ and $u_{2}(x)$ be two distinct solutions of problem (1.2).

Then we have

$$
\int_{\Omega} u_{1} u_{2}\left(u_{1}^{p-1}-u_{2}^{p-1}\right) d x=0
$$

Proof. The proof of this Lemma is merely an application of integration by part, so we omit it here. A similar argument can be found in the proof of Lemma 2.6. 
LEMMA 2.6. Let $\left(u_{1}(x), v_{1}(x)\right)$ and $\left(u_{2}(x), v_{2}(x)\right)$ be two distinct solutions of problem (1.3).

Then we have

$$
\int_{\Omega} u_{1} u_{2}\left(u_{1}^{q-1}-u_{2}^{q-1}\right) d x=\int_{\Omega} v_{1} v_{2}\left(v_{2}^{p-1}-v_{1}^{p-1}\right) d x .
$$

Proof. Since $\left(u_{1}(x), v_{1}(x)\right)$ and $\left(u_{2}(x), v_{2}(x)\right)$ are solutions of problem (1.3), we have

$$
\begin{cases}\Delta u_{1}+v_{1}^{p}=0, & x \in \Omega, \\ \Delta v_{1}+u_{1}^{q}=0, & x \in \Omega, \\ u_{1}, v_{1}>0, & x \in \Omega, \\ B u_{1}=B v_{1}=0, & x \in \partial \Omega,\end{cases}
$$

and

$$
\begin{cases}\Delta u_{2}+v_{2}^{p}=0, & x \in \Omega, \\ \Delta v_{2}+u_{2}^{q}=0, & x \in \Omega, \\ u_{2}, v_{2}>0, & x \in \Omega, \\ B u_{2}=B v_{2}=0, & x \in \partial \Omega .\end{cases}
$$

Accordingly

$$
\begin{aligned}
& \int_{\Omega} v_{2} v_{1}^{p} d x=\int_{\Omega} v_{2}\left(-\Delta u_{1}\right) d x=\int_{\Omega} u_{1}\left(-\Delta v_{2}\right) d x=\int_{\Omega} u_{1} u_{2}^{q} d x \\
& \int_{\Omega} v_{1} v_{2}^{p} d x=\int_{\Omega} v_{1}\left(-\Delta u_{2}\right) d x=\int_{\Omega} u_{2}\left(-\Delta v_{1}\right) d x=\int_{\Omega} u_{2} u_{1}^{q} d x .
\end{aligned}
$$

Combining the above two identities, we conclude the proof of Lemma 2.6.

\section{The proof of Theorems 1.1 and 1.2.}

Proof of Theorem 1.1. Suppose that the conclusion of Theorem 1.1 is false. Then there exists a sequence $\eta_{i} \rightarrow 0$ as $i \rightarrow \infty$ such that the problem

$$
\begin{cases}\Delta u+u^{p}=0, & x \in \Omega, \\ u>0, & x \in \Omega, \\ \frac{\partial u}{\partial v}+\eta_{i} u=0, & x \in \partial \Omega,\end{cases}
$$

has at least two distinct solutions $u_{i}^{1}(x)$ and $u_{i}^{2}(x)$. By Lemma 2.3 and the standard elliptic estimates (see [5]), we know that there exists a positive constant $C$ independent of $\eta_{i}$ such that

$$
\left\|u_{i}^{1}\right\|_{C^{2, \alpha}(\bar{\Omega})},\left\|u_{i}^{2}\right\|_{C^{2, \alpha}(\bar{\Omega})} \leq C .
$$

Hence, up to a subsequence, we may assume that

$$
\begin{array}{ll}
u_{i}^{1}(x) \rightarrow u^{1}(x) & \text { uniformly on } \bar{\Omega} \\
u_{i}^{2}(x) \rightarrow u^{2}(x) & \text { uniformly on } \bar{\Omega} .
\end{array}
$$


Considering the limit in problem (3.1) as $i \rightarrow \infty$, we find that $u^{1}(x)$ and $u^{2}(x)$ are the non-negative solutions of the following problem:

$$
\begin{cases}\Delta u+u^{p}=0, & x \in \Omega, \\ \frac{\partial u}{\partial v}=0, & x \in \partial \Omega .\end{cases}
$$

Consequently, we have $u^{1}(x) \equiv u^{2}(x) \equiv 0$ on $\bar{\Omega}$.

Let $U_{i}(x)=u_{i}^{1}(x)-u_{i}^{2}(x)$. Then $U_{i}(x) \not \equiv 0$ since $u_{i}^{1}(x)$ and $u_{i}^{2}(x)$ are distinct. Thus, we can define

$$
w_{i}(x)=\frac{U_{i}(x)}{\left\|U_{i}\right\|_{L^{\infty}(\bar{\Omega})}} .
$$

It is easy to verify that $w_{i}(x)$ satisfies

$$
\begin{cases}\Delta w_{i}+p a_{i}^{p-1}(x) w_{i}=0, & x \in \Omega, \\ \left\|w_{i}\right\|_{L^{\infty}(\bar{\Omega})}=1, & \\ \frac{\partial w_{i}}{\partial v}+\eta_{i} w_{i}=0, & x \in \partial \Omega,\end{cases}
$$

where $a_{i}(x)$ is a function between $u_{i}^{1}(x)$ and $u_{i}^{2}(x)$.

Noticing that $p a_{i}^{p-1}(x) w_{i}$ are uniformly bounded, it follows from the standard elliptic estimates that there exists a positive constant $C$ independent of $\eta_{i}$ such that $\left\|w_{i}\right\|_{C^{2, \alpha}(\bar{\Omega})} \leq C$. Hence, up to a subsequence, we may assume that

$$
w_{i}(x) \rightarrow w(x) \quad \text { uniformly on } \bar{\Omega} .
$$

Considering the limit in problem (3.3) as $i \rightarrow \infty$, we conclude that $w(x)$ satisfies

$$
\begin{cases}\Delta w=0, & x \in \Omega, \\ \|w\|_{L^{\infty}(\bar{\Omega})}=1, & \\ \frac{\partial w}{\partial v}=0, & x \in \partial \Omega .\end{cases}
$$

This implies that $w(x) \equiv 1$ on $\bar{\Omega}$, or $w(x) \equiv-1$ on $\bar{\Omega}$. Without loss of generality, we may assume that $w(x) \equiv 1$. Then there exists a positive integer $I$ such that

$$
w_{i}(x)>0 \text { for any } i>I \text { and } x \in \bar{\Omega} \text {. }
$$

Accordingly, by the definition of $w_{i}(x)$, we know that

$$
u_{i}^{1}(x)-u_{i}^{2}(x)>0 \text { for any } i>I \text { and } x \in \bar{\Omega} .
$$

This contradicts the conclusion of Lemma 2.5. Thus, we complete the proof of Theorem 1.1. 
Proof of Theorem 1.2. Suppose that the conclusion of Theorem 1.2 is false. Then there exists a sequence $\eta_{i} \rightarrow 0$ as $i \rightarrow \infty$ such that the problem

$$
\begin{cases}\Delta u+v^{p}=0, & x \in \Omega, \\ \Delta v+u^{q}=0, & x \in \Omega, \\ u, v>0, & x \in \Omega, \\ \frac{\partial u}{\partial v}+\eta_{i} u=0, & x \in \partial \Omega, \\ \frac{\partial v}{\partial v}+\eta_{i} v=0, & x \in \partial \Omega,\end{cases}
$$

has at least two distinct solutions $\left(u_{i}^{1}(x), v_{i}^{1}(x)\right)$ and $\left(u_{i}^{2}(x), v_{i}^{2}(x)\right)$. By Lemma 2.4 and the standard elliptic estimates (see [5]), we know that there exists a positive constant $C$ independent of $\eta_{i}$ such that

$$
\left\|u_{i}^{1}\right\|_{C^{2, \alpha}(\bar{\Omega})}+\left\|v_{i}^{1}\right\|_{C^{2, \alpha}(\bar{\Omega})} \leq C,
$$

and

$$
\left\|u_{i}^{2}\right\|_{C^{2, \alpha}(\bar{\Omega})}+\left\|v_{i}^{2}\right\|_{C^{2, \alpha}(\bar{\Omega})} \leq C .
$$

Hence, up to a subsequence, we may assume that

$$
\begin{array}{ll}
u_{i}^{1}(x) \rightarrow u^{1}(x) & \text { uniformly on } \bar{\Omega}, \\
u_{i}^{2}(x) \rightarrow u^{2}(x) & \text { uniformly on } \bar{\Omega}
\end{array}
$$

and

$$
\begin{array}{ll}
v_{i}^{1}(x) \rightarrow v^{1}(x) & \text { uniformly on } \bar{\Omega} \\
v_{i}^{2}(x) \rightarrow v^{2}(x) & \text { uniformly on } \bar{\Omega} .
\end{array}
$$

Considering the limit in problem (3.5) as $i \rightarrow \infty$, we find that $\left(u^{1}(x), v^{1}(x)\right)$ and $\left(u^{2}(x), v^{2}(x)\right)$ are non-negative solutions of the following problem:

$$
\begin{cases}\Delta u+v^{p}=0, & x \in \Omega, \\ \Delta v+u^{q}=0, & x \in \Omega, \\ \frac{\partial u}{\partial v}=\frac{\partial v}{\partial v}=0, & x \in \partial \Omega .\end{cases}
$$

Consequently, we have $u^{1}(x) \equiv u^{2}(x) \equiv v^{1}(x) \equiv v^{2}(x) \equiv 0$ on $\bar{\Omega}$.

Let $U_{i}(x)=u_{i}^{1}(x)-u_{i}^{2}(x)$ and $V_{i}(x)=v_{i}^{1}(x)-v_{i}^{2}(x)$.

Then $\left|U_{i}(x)\right|+\left|V_{i}(x)\right| \not \equiv 0$ since $\left(u_{i}^{1}(x), v_{i}^{1}(x)\right)$ and $\left(u_{i}^{2}(x), v_{i}^{2}(x)\right)$ are distinct. Thus, we can define $w_{i}(x)=U_{i}(x) /\left(\left\|U_{i}\right\|_{L^{\infty}(\bar{\Omega})}+\left\|V_{i}\right\|_{L^{\infty}(\bar{\Omega})}\right)$ and $h_{i}(x)=V_{i}(x) /\left(\left\|U_{i}\right\|_{L^{\infty}(\bar{\Omega})}+\right.$ $\left.\left\|V_{i}\right\|_{L^{\infty}(\bar{\Omega})}\right)$. 
It is easy to verify that $\left(w_{i}(x), h_{i}(x)\right)$ satisfies

$$
\begin{cases}\Delta w_{i}+p a_{i}^{p-1}(x) h_{i}=0, & x \in \Omega, \\ \Delta h_{i}+q b_{i}^{q-1}(x) w_{i}=0, & x \in \Omega, \\ \left\|w_{i}\right\|_{L^{\infty}(\bar{\Omega})}+\left\|h_{i}\right\|_{L^{\infty}(\bar{\Omega})}=1, & \\ \frac{\partial w_{i}}{\partial v}+\eta_{i} w_{i}=0, & x \in \partial \Omega, \\ \frac{\partial h_{i}}{\partial v}+\eta_{i} h_{i}=0, & x \in \partial \Omega,\end{cases}
$$

where $a_{i}(x)$ is a function between $v_{i}^{1}(x)$ and $v_{i}^{2}(x)$ and $b_{i}(x)$ is a function between $u_{i}^{1}(x)$ and $u_{i}^{2}(x)$.

Noticing that $p a_{i}^{p-1}(x) h_{i}$ and $q b_{i}^{q-1}(x) w_{i}$ are uniformly bounded, it follows from the standard elliptic estimates that there exists a positive constant $C$ independent of $\eta_{i}$ such that $\left\|w_{i}\right\|_{C^{2, \alpha}(\bar{\Omega})} \leq C$ and $\left\|h_{i}\right\|_{C^{2, \alpha}(\bar{\Omega})} \leq C$. Hence, up to a subsequence, we may assume that

$$
\begin{aligned}
& w_{i}(x) \rightarrow w(x) \quad \text { uniformly on } \bar{\Omega} . \\
& h_{i}(x) \rightarrow h(x) \quad \text { uniformly on } \bar{\Omega} .
\end{aligned}
$$

Considering the limit in problem (3.7) as $i \rightarrow \infty$, we conclude that $(w(x), h(x))$ satisfies

$$
\begin{cases}\Delta w=0, & x \in \Omega, \\ \Delta h=0, & x \in \Omega, \\ \|w\|_{L^{\infty}(\bar{\Omega})}+\|h\|_{L^{\infty}(\bar{\Omega})}=1, & \\ \frac{\partial w}{\partial v}=\frac{\partial h}{\partial v}=0, & x \in \partial \Omega .\end{cases}
$$

This implies that $w(x) \equiv C_{1}$ and $h(x) \equiv C_{2}$ on $\bar{\Omega}$, where $C_{1}$ and $C_{2}$ are constants. Moreover, $\left|C_{1}\right|+\left|C_{2}\right|=1$. Hence, one of $\left|C_{1}\right|$ and $\left|C_{2}\right|$ should be no less than $1 / 2$. Without loss of generality, we may assume that $\left|C_{1}\right| \geq 1 / 2$. Then $C_{1} \geq 1 / 2$ or $C_{1} \leq$ $-1 / 2$.

If $C_{1} \geq 1 / 2$, then there exists a positive integer $I$ such that

$$
w_{i}(x)>0 \text { for any } i>I \text { and } x \in \bar{\Omega},
$$

from which we can also conclude that

$$
h_{i}(x)>0 \text { for any } i>I \text { and } x \in \bar{\Omega} .
$$

Now, by the definition of $w_{i}(x)$ and $h_{i}$, we know that

$$
u_{i}^{1}(x)-u_{i}^{2}(x)>0 \text { for any } i>I \text { and } x \in \bar{\Omega},
$$

and

$$
v_{i}^{1}(x)-v_{i}^{2}(x)>0 \text { for any } i>I \text { and } x \in \bar{\Omega} .
$$

This contradicts the conclusion of Lemma 2.6. 
If $C_{1} \leq-1 / 2$, by a similar argument, we may conclude that there exists a positive integer $I$ such that

$$
u_{i}^{1}(x)-u_{i}^{2}(x)<0 \text { for any } i>I \text { and } x \in \bar{\Omega},
$$

and

$$
v_{i}^{1}(x)-v_{i}^{2}(x)<0 \text { for any } i>I \text { and } x \in \bar{\Omega} .
$$

This also contradicts the conclusion of Lemma 2.6. Thus, we complete the proof of Theorem 1.2.

\section{REFERENCES}

1. W. M. Ni and R. D. Nussbaum, Uniqueness and non-uniqueness for positive solutions of $\Delta u+f(r, u)=0$, Commun. Pure Appl. Math. 38(1985), 67-108.

2. Q. Y. Dai, Entire positive solutions for inhomogeneous semilinear elliptic systems, Glasgow Math. J. 47(2005), 97-114.

3. Q. Y. Dai, Y. X. Fu and Y. G. Gu, Uniqueness of positive solutions of semilinear elliptic equations, Sci. China A 50 (2007), 1141-1156.

4. B. Gidas and J. Spruck, A priori bounds for positive solutions of semilinear elliptic equations, Commun. Part. Differ. Equ. 6(1981), 883-901.

5. D. Gilbarg and N. S. Trudinger, Elliptic partial differential equations of second order (Springer-Velag, Berlin, Germany, 2001).

6. L. Damascelli, M. Grossi and F. Pacella, Qualitative properties of positive solutions of semilinear equations in symmetric domains via maximum principle, Ann. Inst. Henri Poincare Analse non lineaire 16(1999), 631-652.

7. E. N. Dancer, The effect of domain shape on the number of positive solutions of certain weakly nonlinear equations, J. Differ. Equ. 74(1988), 120-156.

8. E. N. Dancer, On the influence of domain shape on the existence of large solutions of some superlinear problems, Math. Ann. 285(1989), 647-669.

9. X. J. Wang, Neumann problems of semilinear elliptic equations involving critical Sobolev exponents, J. Differ. Equ. 93(1991), 283-310.

10. H. Zou, On the effect of domain geometry on uniqueness of positive solutions of $\Delta u+u^{p}=0$, Ann. Scuola Norm. Super. Pisa 21(1994), 343-356.

11. H. Zou, A priori estimates for semilinear elliptic system without variational structure and their application, Math. Ann. 323(2002), 713-735.

12. D. de Figueiredo and P. Felmer, A Liouville type theorem for systems, Ann. Scuola Norm. Super. Pisa 21(1994), 387-397.

13. J. C. Wei and L. Q. Zhang, On the effect of the domain shape on the existence of large solutions of some superlinear problems, Preprint (2006).

14. H. Berestycki, L. Nirenberg and S. N. S. Varadhan, The principle eigenvalues and maximum principle for second order elliptic operators in general domains, Commun. Pure Appl. Math. 47(1994), 47-92.

15. L. Q. Zhang, Uniqueness of positive solutions of $\Delta u+u^{p}=0$ in a convex domain in $R^{2}$, Preprint (1992).

16. L. Q. Zhang, Uniqueness of positive solutions of $\Delta u+u+u^{p}=0$ in a finite ball, Commun. Part. Differ. Equ. 47(1992), 1141-1164.

17. C. S. Lin, Uniqueness of solutions minimizing the functional $\int_{\Omega}|\nabla u|^{2} /\left(\int_{\Omega}|u|^{p+1}\right)^{2 /(p+1)}$ in $R^{2}$, Manuscript Math. 84(1) (1994), 13-19. 